\title{
Kernos
}

Revue internationale et pluridisciplinaire de religion grecque antique

6 | 1993

Varia

\section{Chronique des fouilles}

Ioannis Loucas et Éveline Loucas-Durie

\section{QpenEdition \\ Journals}

\section{Édition électronique}

URL : http://journals.openedition.org/kernos/558

DOI : $10.4000 /$ kernos.558

ISSN : 2034-7871

\section{Éditeur}

Centre international d'étude de la religion grecque antique

Édition imprimée

Date de publication : 1 janvier 1993

Pagination : 343-359

ISSN : 0776-3824

\section{Référence électronique}

Ioannis Loucas et Éveline Loucas-Durie, «Chronique des fouilles », Kernos [En ligne], 6| 1993, mis en ligne le 07 avril 2011, consulté le 16 septembre 2020. URL : http://journals.openedition.org/kernos/ 558 
Kernos, 6 (1993), p. 343-359.

\section{CHRONIQUE DES FOUILLES}

par Ioannis LOUCAS et Éveline LOUCAS-DURIE

\section{A. Activités de la Société archéologique d'Athènes en 1991}

Informations non communiquées.

\section{B. Activités des Écoles et Instituts d'archéologie étrangers en 1992}

\section{Activités de l'École américaine d'Études classiques}

Athènes, agora (T. Leslie Shear, Jr., J. Mck. Camp, II)

Les travaux de la campagne 1992 ont porté sur deux secteurs : le premier, au nord de la place de l'Agora, le long de la voie qui conduit hors de l'Agora en direction nord-ouest (ce qui correspond actuellement au côté sud de la rue Hastings) et le second, à l'angle nord-ouest de l'Agora (aujourd'hui le côté nord de la rue Hadrianou). Ont été mis au jour un puits dont le premier usage remonte au IIIe s. apr. J.-C. et surtout des vestiges d'époque byzantine.

\section{Halai (J.E. Coleman)}

Les travaux sur l'acropole d'Halai ont permis de distinguer les principales époques d'occupation du site : début ou fin du néolithique ancien, périodes archaïque, hellénistique, romaine tardive. À signaler l'abondance de trouvailles attestant une activité intensive aux Ve et VIe s., auxquelles s'ajoute la découverte de deux tombes de cette période. La première était la sépulture d'un enfant, inhumé sans offrandes, recouverte de tuiles. La deuxième était une tombe construite à voûte incarnée, de toute évidence plus importante. Bien qu'elle ait été profanée dès l'antiquité, le fouilleur a pu estimer le nombre d'inhumations d'après les ossements épars à au moins seize. Dans le couloir et dans la salle principale de la sépulture ont été repérées respectivement quinze et deux lampes du Ve ou du VIe s., presque toutes décorées de symboles chrétiens. Selon le fouilleur, ces sépultures, exhumées dans le quartier $\mathrm{H}$, seraient postérieures à l'habitat, tout au moins dans la moitié nord-ouest de l'acropole, et devraient être mises en rapport avec le sanctuaire paléochrétien du quartier $\mathrm{G}$, distant de $30 \mathrm{~m}$ environ, lequel serait aussi postérieur à l'habitat sur l'acropole. Autrement dit, 
souligne le fouilleur, le culte chrétien n'aurait commencé qu'après l'abandon de la ville. Afin de préciser la datation de l'église, on a procédé à un sondage dans la nef centrale et dans l'aile nord de l'abside. Une mosaïque de pavement en petites tessères de matériau artificiel et de pierres naturelles a été repérée. Les premiers examens conduisent à la dater au VIe $\mathbf{s}$. Les sujets de trois panneaux figurés ont été identifiés : des oiseaux dans un bol, un cygne, un aigle avec un halo.

\section{Isthmia (E.R. Gebhard - University of Chicago)}

Étude du matériel et de l'architecture en vue de la préparation de la publication finale. Le temple archaïque a donné de la céramique attique, en grande partie détruite par un incendie, daté ainsi aux environs de 470 av. J.-C. Le grand nombre d'amphores brûlées sur leur surface interne retrouvé dans le sèkos montre qu'à l'époque de l'incendie, de l'huile, et peut-être du vin, y était entreposée, tandis que des aryballes, des alabastres, en forte concentration, et d'autres objets en céramique de qualité exceptionnelle, ainsi que des bijoux, des monnaies et des objets importés étaient conservés dans le pronaos.

Les vases de la période de 700 à 550 av. J.-C. sont des coupes, principalement des kotyles, dont les types se suivent depuis la deuxième moitié du VIIIe $s$. La céramique miniature se limite à de petites corbeilles. À la fin du VIe et au Ve s., des coupes à vernis noir et à une anse (dont une porte l'inscription «kothon» et une autre "consacré à Poséidon») constituent probablement des offrandes, déposées par des soldats et des voyageurs.

Quant à l'étude architecturale, elle a révélé que les fragments travaillés de poros trouvés dans la fouille de la terrasse orientale en 1989 proviennent de l'exécution des cannelures des colonnes du Temple Classique. Elle a aussi permis de préciser l'origine de membres architecturaux dans le côté nord du temenos: ils proviennent d'un petit édifice dorique, une porte sans doute. Des fragments du Temple Classique de Poséidon ont été retrouvés, de même qu'une inscription publique de 220 av. J.-C. dans le dépôt d'où proviennent les blocs doriques. Selon le fouilleur, ceci correspondrait à un nettoyage des lieux après des dégâts, causés peut-être par une action guerrière, vers 200 av. J.-C. D'autres fragments en grand nombre et des colonnes du Temple Classique de Poséidon ont été retrouvés dans des dépôts des environs de 100 av. J.-C., associés à la construction du premier temenos romain.

La sculpture de marbre non inclue dans Isthmia IV compte presque 300 fragments qui permettent finalement de reconstituer un ensemble de huit statues - portraits, plus grandes que nature du IIe apr. J.-C. 
Elles devaient s'élever dans le Palaimonion d'Antonin et représenter des personnages importants.

Isthmia (T.E. Gregory - University of Ohio)

Continuation des travaux de conservation de la mosaïque monochrome des thermes romains, découverts en 1976 et retirée en 1990.

Des recherches ont été menées aussi au sud des thermes dans une région fouillée en 1978 et en 1980 et qui avait été décrite comme un dépôt sud de lampes.

Corinthe (Charles K. Williams II - American School of Classical Studies)

Les travaux de fouille ont porté sur la région située au sud du musée de l'ancienne Corinthe et ont mis au jour plus de $1400 \mathrm{~m}^{2}$ de la ville franque.

Némée (St. G. Miller - University of California at Berkeley)

L'essentiel des activités s'est concentré sur la préparation de la publication définitive des précédentes campagnes. Un premier tome est déjà paru : D.E. BIRGE, L.H. KRAYNAC, S.G. MILLER, Nemea I : Topographical and Architectural Studies; the Sacred Square, the Xenon, and the Bath, Berkeley, 1992.

Les travaux de l'été '92 concernaient la préparation du tome sur le Stade. Les quelques travaux effectués dans le sanctuaire visaient à protéger les vestiges des dégradations causées par les intempéries.

\section{Pylos}

"Pylos Regional Archeological Project» (Jack L. Davis, University of Illinois at Chicago - American School of Classical Studies).

Ce projet, dont la première saison de recherche de surface s'est déroulée l'été dernier, vise à relever systématiquement les traces d'activités passées dans un territoire de $250 \mathrm{~km}^{2}$ au sud-ouest de la Messénie dont le Palais de Nestor à Pylos constitue le centre. Un des principaux objectifs du projet est de reconstituer et d'interpréter les changements de l'époque préhistorique dans les structures d'habitat et dans l'usage de la terre avant l'apparition du palais, durant son fonctionnement en tant que centre et après son déclin. Un deuxième objectif consiste à reconstituer les structures d'habitat aux périodes historiques, avant, pendant, et après la domination spartiate sur la Messénie. 
Durant cette saison, l'enquête de surface a couvert environ $12 \mathrm{~km}^{2}$ et a fourni d'importantes informations concernant l'étendue de l'habitat autour du palais. Dix-huit autres sites ont fait l'objet de recherches, parmi lesquels figurent des cimetières d'époques préhistorique et historique.

«Minnesota Pylos Project» (F.A. Cooper - M.C. Nelson, University of Minnesota).

Continuation des travaux de nettoyage en vue de l'élaboration d'un plan exact des murs, dans les salles 89 et 90 dans la partie occidentale du palais et dans l'Aire 103, à l'est et au sud du Magasin de vin. Dans cette dernière région est apparue une succession de sols et de murs, allant de l'époque prépalatiale à l'époque postpalatiale, qui n'avait guère attiré l'attention de Blegen. Les fouilleurs en ont établi une chronologie relative provisoire en sept phases.

\section{Samothrace}

Sanctuaire des Grands Dieux (J.R. McCredie, Institute of Fine Arts, New York University)

Parmi les travaux effectués dans les réserves du musée et sur le site, dont un important travail d'inventaire, les plus importants sont:

- Réétude des éléments appartenant au Néôrion, sur la colline occidentale et reconstruction détaillée de cet édifice, laquelle est presque achevée.

- Étude des figurines en terre provenant des remblais du propylée de Ptolémée II.

- Inventaire des blocs de marbre de l'ex-voto de Philippe III et d'Alexandre IV, sur la colline orientale, en vue de la publication. Une nouvelle édition du «Guide» est en préparation.

\section{Crète}

a. Gournia (C. Davaras, V. Watrous)

Saison d'activité intense en raison du projet d'une nouvelle route nationale Gournia-Pakheia Ammos, pour le tracé de laquelle, d'ailleurs, V. Watrous et D. Haggis ont été invités par l'Éphorie à conseiller les autorités préfectorales, afin d'épargner des sites importants. Dix-neuf sites ont été enregistrés durant la campagne, dont celui des tombes d'Altsomouroi qui comprend des tombes à chambre et des sépultures en pithos sur les versants de la crête montagneuse et sur son sommet. Elles se situent chronologiquement entre le Minoen Moyen I et le Minoen Récent I. 


\section{b. Grevena (Nancy C. Wilkie)}

Les objectifs de la saison étaient l'étude de la céramique et d'autres trouvailles et celle des profils de sol dans un certain nombre d'endroits par des géologues.

\section{c. Mokhlos (C. Davaras, J. Soles)}

Les fouilles menées en commun par l'Éphorie et l'École américaine se sont poursuivies : 1) sur l'île de Mokhlos, dans la région de l'habitat minoen; 2) sur la route qui conduit de Mokhlos à Limanari, en direction du sud-ouest, où a été repérée une extension de l'habitat du Minoen Récent IB; 3) au lieu dit Khalinomouri, à l'extrémité orientale de la plaine côtière de Mokhlos, où il y a une installation agricole du Minoen Récent IB.

Les travaux sur l'île se sont concentrés sur le grand édifice en appareil isodome repéré l'été dernier. Les façades $E$ et $S$ ont été dégagées, ainsi que l'entrée principale qui conduit au niveau du deuxième étage, depuis la rue qui longe le côté oriental de l'édifice. Elle mène par un vestibule à un polythuron, pavé de plaques de schiste vert, qui donne accès aux différentes parties de l'édifice, d'un plan complexe, à trois étages. Un réservoir (impluvium) flanqué de deux colonnes et d'un kernos s'appuyait au mur nord du polythuron et sa place suggère aux fouilleurs qu'il constituait un élément majeur de la pièce et même de l'édifice général. Cet édifice est la construction la plus grande et la plus imposante de Mokhlos, la seule en appareil isodome. Le dégagement des angles $\mathrm{NE}$ et SO de l'édifice permet de juger de son étendue. Tout ceci amène les fouilleurs à l'identifier au centre administratif et religieux de l'habitat du Minoen Récent IB.

Sur la route conduisant à Limanari, la fouille a révélé deux édifices à fonction industrielle (l'un était centre de travail du bronze et de fabrication de vases en pierre, l'autre, un atelier de céramique) qui semblent disposer chacun d'un petit sanctuaire. L'un consistait en un sanctuaire à banquette, l'autre en une salle, partiellement fouillée, où un ex-voto en forme de pied a été retrouvé. L'un et l'autre édifice se prolongent dans le champ contigu, au sud-ouest. De l'avis des fouilleurs, il y avait des pièces importantes, comprenant celle à l'ex-voto et une autre, partiellement fouillée, qui aurait pu servir de trésor au sanctuaire à banquette. 


\section{d. Pseira (Ph. P. Betancourt, C. Davaras)}

Les travaux ont consisté en l'étude architecturale des vestiges sur le site et en l'étude des trouvailles conservées à Mokhlos.

e. Kavousi (W.D.E. Couson, G.C. Gesell, L.P. Day)

Les travaux ont porté sur l'habitat inférieur à Vronda, et sur l'habitat supérieur de Kastro.

\section{f. Kommos (J.W. Shaw - University of Toronto - Royal Ontario Museum)}

La 17e saison de fouille à Kommos a vu se poursuivre la fouille de la cité minoenne et celle du sanctuaire d'époque grecque. De la première, ce sont la Maison X et deux imposants édifices civiques, $\mathrm{T}$ du MR I et $\mathrm{P}$ du MR III, qui ont été dégagés dans leur majeure partie. La maison X a été occupée du MR IA au MR IIIA2 et ses limites nord, sud et ouest ont été définies. La pièce X1 et l'espace X8 ont donné différents fragments de fresques. Le décor de la pièce $\mathrm{X} 1$ comporte des lys blancs sur un fond ocre dans un paysage rocheux. La décoration de fresque dont on retrouve des traces dans d'autres pièces encore, témoigne, aux yeux du fouilleur, de la haute importance de cette habitation durant son épanouissement au MR I.

L'édifice P du MR III, le plus grand édifice connu de cette époque (il s'étend sur $38,51 \mathrm{~m} \mathrm{E}-\mathrm{O}$ et $39,60 \mathrm{~m} \mathrm{~N}$-S) est constitué d'au moins six galeries couvertes qui donnent, à l'ouest, sur une cour, tandis qu'à l'est elles sont totalement fermées par une façade monumentale de grande épaisseur. Le nettoyage de la Galerie 3 dans sa totalité a révélé qu'elle n'était pourvue d'aucune porte sur les côtés nord et sud, qu'elle n'était accessible que par l'ouest, qu'elle était haute de $4 \mathrm{~m}$ environ. Selon le fouilleur, il est probable que ces galeries abritaient les bateaux durant les mois où ils ne naviguaient pas. L'édifice semble avoir connu plusieurs utilisations, dont la dernière de l'âge du Bronze est située au MR IIIB et semble avoir été interrompue par un incendie.

L'édifice $\mathrm{T}$, déjà étudié partiellement à l'occasion des campagnes précédentes, est maintenant connu dans sa distribution générale : des murs de façade extérieurs en appareil isodome, des ailes au nord, à l'est et au sud, qui donnent sur une cour centrale, caractéristiques, souligne le fouilleur, qui lui confêrent de toute évidence un aspect de palais, s'il ne s'agit pas d'un véritable palais. Cependant, à la différence de ce genre d'édifice, l'édifice $\mathbf{T}$ présente un plan plus régulier et est plus fermé sur l'extérieur, comme si la question de sécurité avait été prioritaire. Quant à l'intérieur des salles, on regrette qu'il ne soit pas mieux 
conservé. Il semble qu'il y avait aussi un deuxième étage. Sous l'aile orientale de l'édifice $\mathbf{T}$ sont apparus des vestiges architecturaux importants du MM, provenant d'un ou de plusieurs grands édifices. Le sanctuaire d'époque grecque, plus précisément les zones au sud et à l'est ont fait l'objet de travaux : fin du dégagement de l'édifice $F$ du Ve $s$. av. J.$\mathrm{C}$., dégagement des vestiges d'un édifice du géométrique moyen $-\mathrm{Z}-$ contemporain du temple $\mathrm{B}$, dans la galerie 3 , découverte d'un bloc carré, probablement la base d'un monument hellénistique, contemporain du temple C.

\section{Activités de l'École française d'Athènes \\ Delphes}

a. Secteur du Char des Rhodiens (J.-M. Luce)

Poursuite du dégagement des constructions antérieures au Char, plus précisément d'une maison d'époque géométrique, nommée maison jaune en raison de la couleur de son sol. Au contraire de la "maison rouge» dont la destruction est datée entre 585 et 575 av. J.-C., la maison jaune ne s'inscrit pas dans l'axe du péribole et de la plupart des monuments du site mais dans un axe nord-est/sud-ouest. Il en va de même

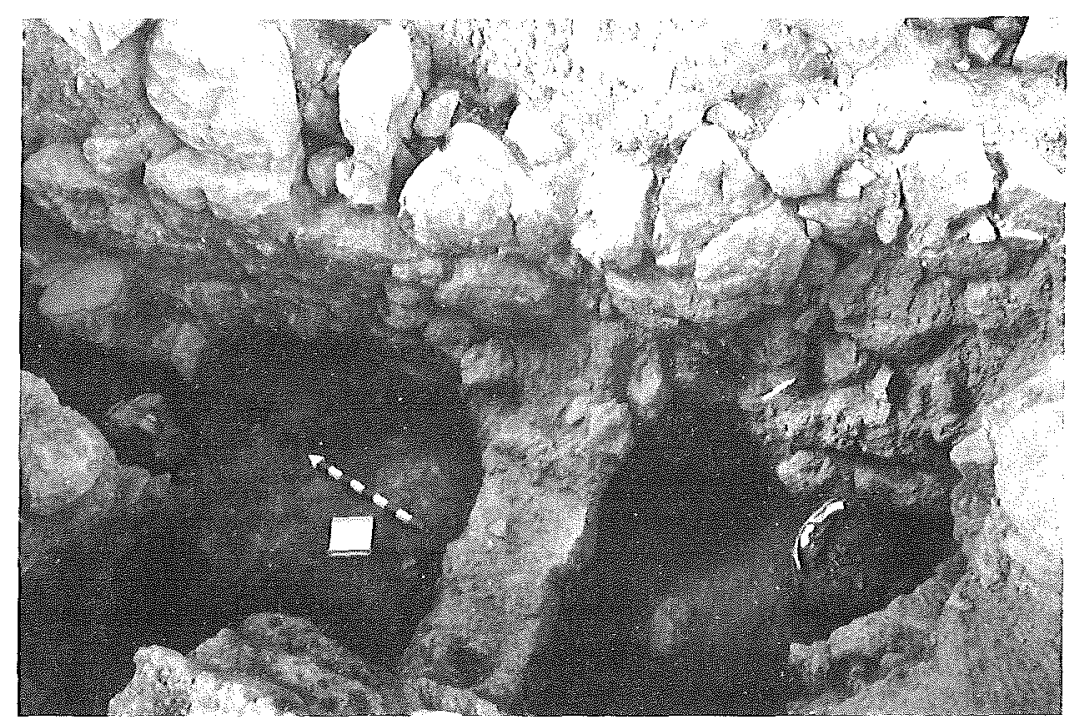

Fig. 1 : Delphes. Salles orientales de la maison noire : vases de stockage en place (photographie École française d'Athènes) 
pour une construction antérieure, découverte sous la maison jaune, et dénommée "maison noire" en raison des traces de destruction par incendie.

Le plan de la maison jaune comporte deux grandes pièces donnant chacune sur une pièce plus étroite située au sud, distribution qui s'est réalisée au moins en deux temps. Plusieurs couches très fines d'argile jaune (d'un usage commun à Delphes) et une seule constituée (assez exceptionnellement) d'enduit de chaux en forment les différents sols et suivent une pente très sensible vers l'ouest. Le dernier sol est daté du dernier quart du VIIIe s. par le matériel au sein duquel un fragment de skyphos de la classe de Thapsos se signale par un essai de polychromie, des lignes rouges venant en effet souligner les lignes noires. Le plan de la maison noire comporte au moins deux pièces alignées, sans communication entre elles, et a été remanié dans un second temps : la pièce la plus à l'ouest a été partagée en deux par une paroi intérieure. Le matériel recueilli se compose de vases de stockage provenant des pièces 2 et 3 (fig. 1-2) et de vaisselle de table et de vases de cuisine, provenant des différents sols de la pièce 1 , ce qui indique, aux yeux du fouilleur, une spécialisation des pièces.

La maison noire a connu plusieurs destructions dont la première a été causée par un incendie vers le milieu du VIIIe s. D'autres couches d'incendie, de la même épaisseur et de date approximativement identique, suggèrent au fouilleur «qu'un seul grand incendie a détruit toute une partie du village vers le milieu du VIIIe s."

Un grand mur de soutènement, auquel s'adossait la maison noire et que la maison jaune a réutilisé et élevé, a maintenu les terres de la terrasse supérieure où l'on retrouve les traces d'incendie observées sur la terrasse inférieure, mais environ $2,30 \mathrm{~m}$ plus haut, sur un sol d'argile jaune. Au-dessous, sous une succession de sols, de couches de destruction et de remblais du IXe s., un petit four en terre, circulaire, a été fouillé (fig. 3). Il était rempli de cendres, de fragments d'os, de graines et de noyaux d'olives, et de céramique de la fin du Xe s. Si la datation se confirme, souligne le fouilleur, il attestera l'existence d'une occupation domestique dès cette époque à Delphes. À ces couches protogéométriques succèdent immédiatement des couches mycéniennes de la fin du XIIIe s. 


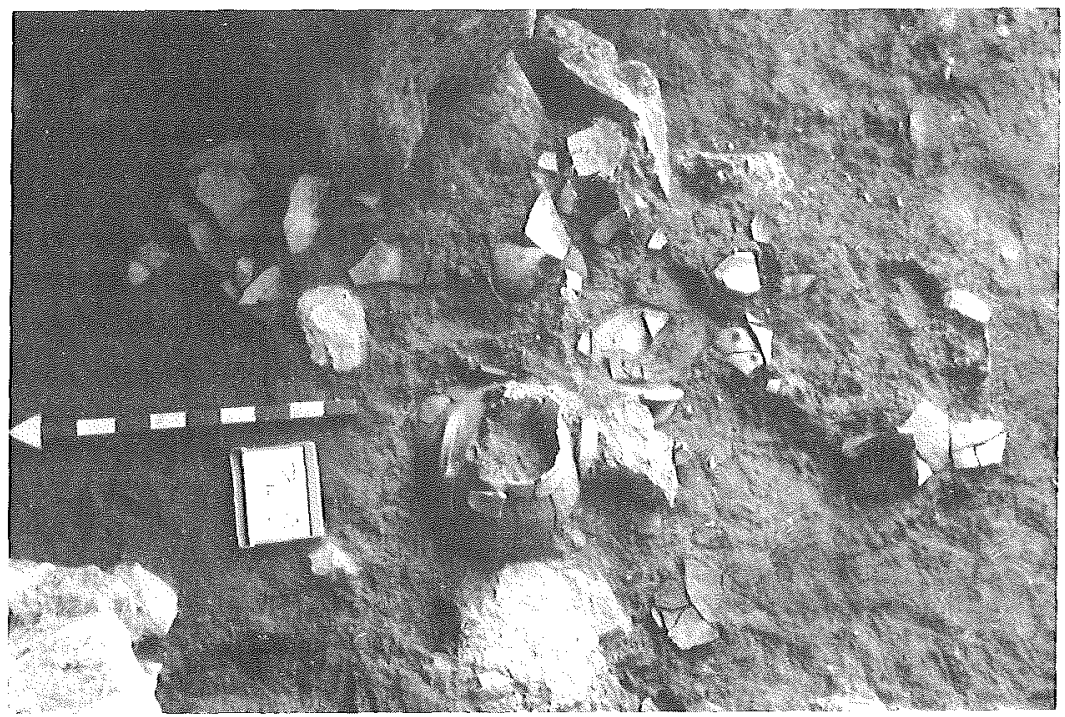

Fig. 2: Delphes. Le dernier sol de la maison noire, salle occidentale (photographie École française d'Athènes)

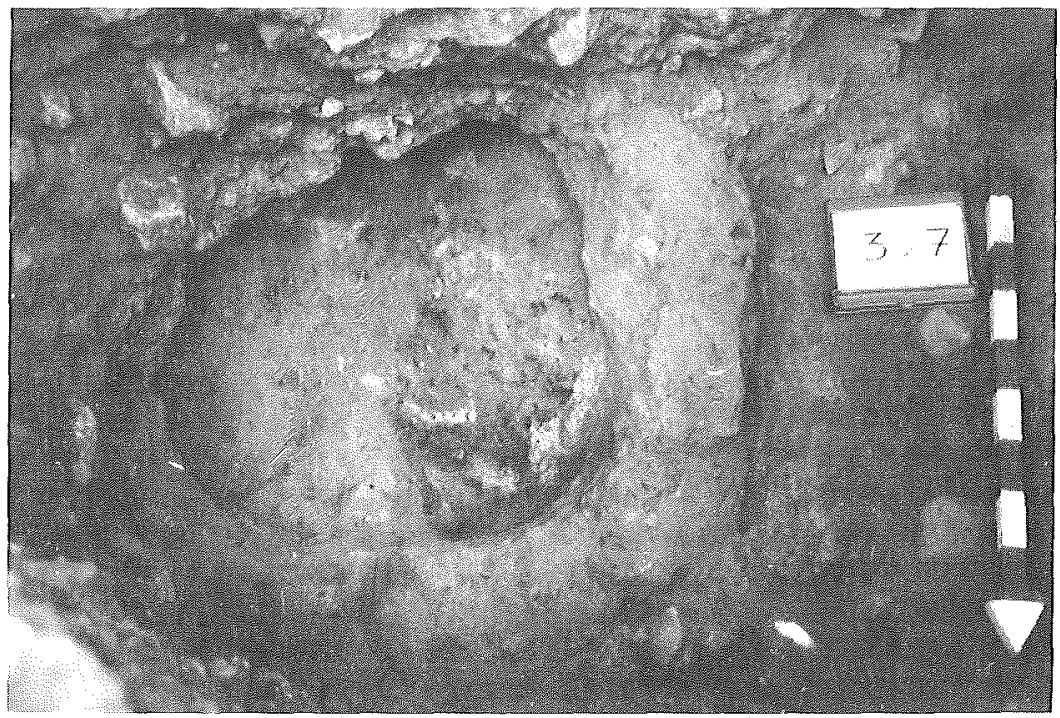

Fig. 3: Delphes. Four du Xe s. av. J.-C. (?) (photographie École française d'Athènes) 


\section{b. Secteur de l'Agora romaine et de la Villa Sud-Est (V. Déroche, P. Petridis)}

Dans le premier secteur, trois sondages ont été ouverts. Le premier a exploré la tranchée de fondation du «mur oblique», livrant de la céramique du Haut Empire. Le deuxième prolongeait le sondage originel dans l'angle intérieur $\mathrm{NE}$ de l'Agora. Le troisième visait à dégager le grand remblai de la boutique murée contiguë à la niche $\mathrm{E}$ de l'Agora. Au fond de ce remblai, les fouilleurs ont rencontré des niveaux d'occupation et d'abandon indiquant une première utilisation comme atelier de verrier, suivie d'une seconde occupation puis d'un abandon et enfin d'un remblaiement général. L'ensemble est situé entre le IVe s. et le Haut Empire, en l'attente d'une chronologie plus affinée fondée sur l'analyse des monnaies.

Dans la "Villa du sud-est» (ex-«Thermes du sud»), dégagement de la pièce apparue au sud de la citerne, contenant une tombe. Un niveau d'occupation, celui de la maison romaine, marqué par un sol de chaux, a pu être précisé au Ve s. Un niveau d'occupation grecque d'une habitation a été repéré.

Enfin, vers l'extrémité $O$ du bâtiment, l'extension de la fouille en direction de la berme du Decauville et de la salle à abside que cette dernière bloquait a permis de dégager un four à céramique installé contre le mur E de l'abside qui, selon les fouilleurs, soit illustre la dégradation de l'habitat urbain à l'extrême fin de l'antiquité, soit, plutôt, est un des témoignages de la réoccupation méso-byzantantine de Delphes.

\section{Thasos}

a. Secteur du Rempart et du Sactuaire d'Athéna (Y. Grandjean, F. Salviat)

Continuation de l'étude du rempart dans la région de l'Acropole en vue de la publication d'ensemble, et plus spécialement en bordure du sanctuaire d'Athéna, et dans la dépression entre ce sanctuaire et le Pythion. L'étude particulière d'un segment de la fortification, en carreaux et en boutisses, à proximité de la fondation du temple d'Athéna révèle que des blocs de la krépis de cet édifice y ont été remployés (fig. 4). Ces blocs et ceux de même nature pris dans la forteresse médiévale permettent aux fouilleurs de restituer au temple d'Athéna, construit au début du Ve s. av. J.-C., trois assises du socle. 


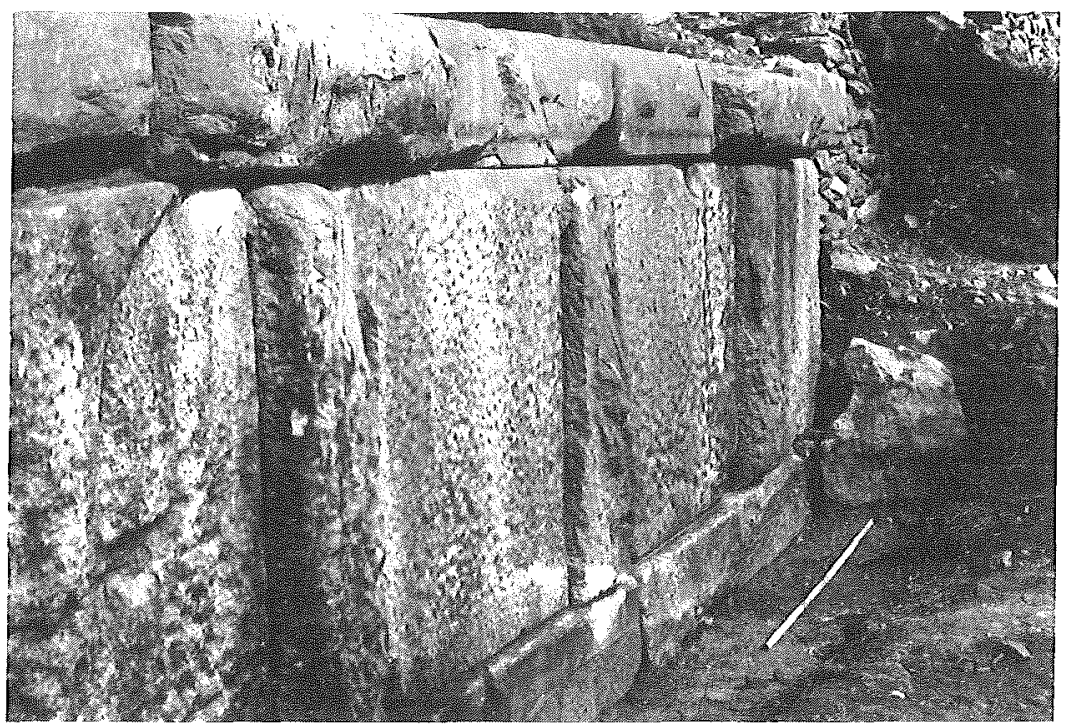

Fig. 4: Section du rempart de Thasos à proximité du temple d'Athéna (photographie de l'École française d'Athènes)

b. Abords nord-est de l'Agora (terrain Valma (F. Blondé, J.-Y. Marc, A. Muller, O. Mulliez)

Reprise des fouilles sous forme de sondages ponctuels en vue de la publication d'ensemble. Dans un des sondages effectués aux abords du Passage des Théores, plus précisément dans le sondage $X$ ouvert au sud du Passage, les fouilleurs ont découvert un tertre artificiel constitué d'une succession de lits de terres hétérogènes, présentant une forte déclivité vers le sud, semblable à celle des plaques de gneiss qui le recouvraient (fig. 5). Le haut du tertre ayant été arasé dès avant la construction du Passage des Théores, il a été facile d'en fouiller l'intérieur sur une petite surface. La présence d'un fragment d'occiput d'enfant parmi un matériel peu abondant permet aux fouilleurs d'interpréter ce tertre comme un tumulus, hypothèse que, de l'avis des fouilleurs, seule une fouille plus étendue permettra de confirmer éventuellement. 


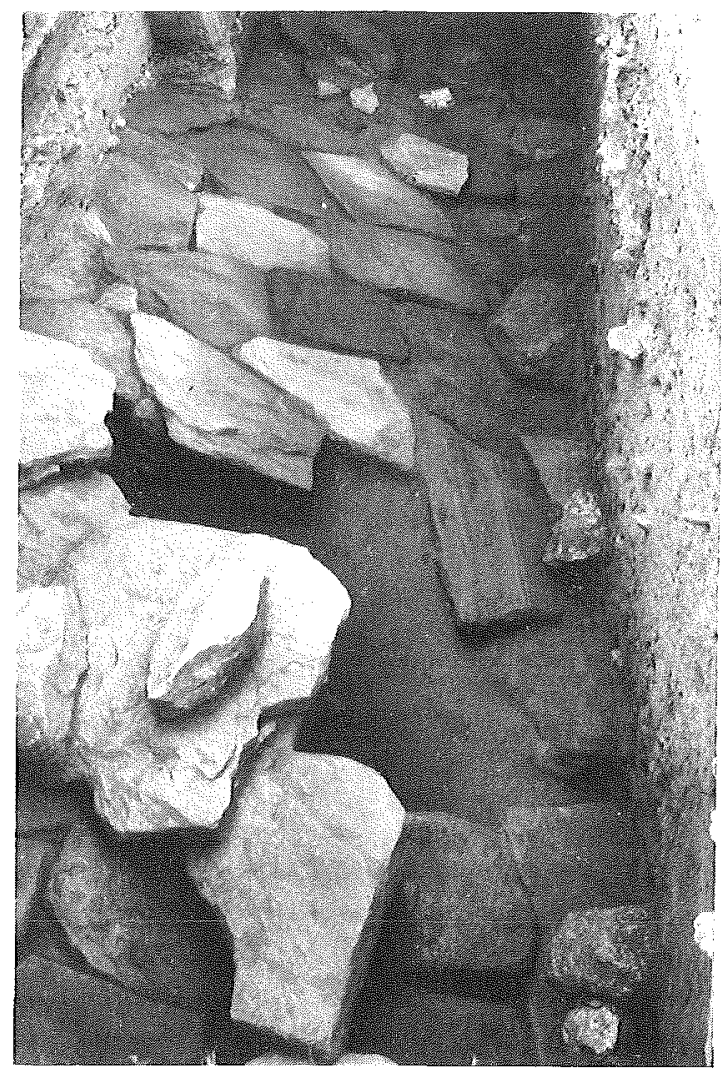

Fig. 5 : Thasos, terrain Valma. Sondage X; vue d'ensemble, vers l'Est. Les plaques de gneiss sur le tertre.

Thessalie. Prospection dans les Chassia (L. Darmezin)

Cette dernière campagne du programme visait à revoir certains sites au nord et au nord-ouest de Kalambaka, dans le nome de Trikala. $\mathrm{Au}$ lieu-dit Loutro qui correspond au toponyme Palaiokastro (à environ $2 \mathrm{~km}$ au nord et en contre-bas de Gavros) des traces d'occupation aux époques hellénistique et romaine ont été repérées. Ce sont essentiellement de la céramique, à quoi s'ajoute une dizaine de blocs parallélipipédiques, en calcaire blanc, longs de $2 \mathrm{~m}$ environ, à face bossée, dont l'un porte des traces de scellement, amassés en bordure d'un champ. Selon L. Darmezin, il pourrait s'agir d'un «habitat ouvert, établi autour ou au voisinage d'un bâtiment construit avec soin, peut-être un petit sanctuaire». 


\section{Malia}

\section{a. Palais (O. Pelon)}

Les travaux de la campagne de 1992 se situaient au nord de l'actuelle cour centrale dans le prolongement des recherches menées en 1990 à l'intérieur et aux alentours de la salle à piliers IX 2 . Il en résulte que les phases proto- et néo-palatiale de la salle à piliers ont été précédées d'un bâtiment important d'une orientation semblable à celle de l'édifice postérieur.

\section{b. Les abords nord-est du palais (C. Baurain, P. Darcque)}

Reprise des travaux interrompus depuis la dernière campagne de 1985. La campagne de 1992 avait pour objectif de compléter les informations architecturales et stratigraphiques sur la zone s'étendant à l'est des quartiers XXV-XXVII du palais. Elle a contribué aussi à clarifier les dispositifs d'accès à l'entrée nord-est du palais; celle-ci a été précédée, assez tôt dans l'époque néopalatiale, d'un porche à trois colonnes, qui donne vers l'est sur un espace à ciel ouvert, lui-même bordé au sud par une esplanade empierrée. La base sud du porche s'articule très nettement avec l'esplanade, et un aménagement semblable se laisse reconnaître à la base nord du porche, malgré les remaniements successifs tout au long de l'époque néo-palatiale.

\section{Activités de l'École italienne d'archéologie}

\section{Sites de Crète}

\section{a. Gortyne (Antonino di Vita)}

Les travaux dans la ville basse, au Prétoire, ont mis au jour l'ensemble des colonnes en granit de Troade qui constituaient la façade majestueuse du portique devant le temple des augusti theoi.

La fouille d'une importante zone au pied de la colline de Prophitis Ilias a livré un monceau de figurines en terre cuite et de petits vases, datant principalement du IIe-Ier s. av. J.-C. et provenant d'un sanctuaire de Déméter situé à proximité, dont l'existence avait déjà été remarquée par Karo au début du siècle. Le matériel abondant mais fragmentaire dans sa majeure partie comprend principalement des figurines de dédicants avec un porcelet. Le fouilleur souligne que la découverte est notable en raison de sa situation en dehors de la ville et à proximité d'une fontaine du sanctuaire de Déméter de Gortyne.

À l'est du Gymnase actuel quelques sépultures de l'époque hellénistique, pauvres en mobilier, ont été découvertes. 
Un important travail d'étude et de conservation du matériel exhumé lors des campagnes précédentes (1989-1991) complétait les activités de fouille.

b. Mitropolis (Antonino di Vita)

Pour la deuxième année, des fouilles ont été effectuées sur le site de la basilique paléo-chrétienne.

\section{c. Haghia Triada (Vincenzo La Rosa)}

Les travaux avaient pour objet le secteur NE occupé par deux constructions de la période néo-palatiale, dénommés "tomba degli Ori» et "Complesso della Mazza di Breccia», et plus particulièrement la région SE de ce dernier édifice. Il s'agissait surtout d'achever d'explorer des tranchées ouvertes en 1991.

\section{d. Phaistos (Vincenzo La Rosa)}

Ouverture de sondages limités dans la région dudit temple grec pour en préciser la chronologie. Les tessons de la fin du VIIIe et du début du VIIe s. av. J.-C. retrouvés dans les trous de fondation constitueraient bien, d'après un premier examen, un terminus ad quem pour la construction de l'édifice. À une phase ultérieure, peut-être hellénistique, le vestibule et la façade de temple ont été reconstruits.

Lemnos. Poliokhni (Alberto Benvenuti)

Prolongation du sondage A ouvert en 1988 au sud de la porte d'entrée de la ville et ouverture d'un sondage M, permettant d'étudier l'extension de la ville à différentes phases situées seulement pour l'instant en termes de chronologie relative. L'ouverture d'un sondage $\mathrm{N}$ a permis de poursuivre l'étude de la région autour du site archéologique, inaugurée en 1991 par une prospection géo-électrique et géo-magnétique ainsi que par un relevé d'échantillons. On a ainsi reconnu des traces d'activités humaines de l'époque préhistorique (de la céramique) près du poste de garde.

\section{Activités de l'Institut archéologique autrichien}

Loussoi. Quartier d'habitation de Phournoi (V. Mitsopoulos-Leon)

Continuation de la fouille des bâtiments du secteur nord de la terrasse inférieure. Dégagement de la partie occidentale de la pièce au foyer et du foyer lui-même. Ce dernier, en terre, est de forme carrée et se compose de quatre carrés longs d'un mètre chacun. Son état actuel atteste d'une deuxième phase d'utilisation lors de laquelle il semble que 
seule la moitié du foyer ait servi. Une épaisse couche de destruction comprenant des tuiles recouvrait le foyer.

À l'ouest de la pièce avec foyer, dégagement d'une pièce spécialement aménagée en salle de bains avec baignoire et cuvette construites, qui ont cependant été enlevées lors d'une deuxième phase d'occupation. À cette deuxième phase correspond sans doute la nouvelle habitation construite vers l'ouest, dont deux pièces ont été fouillées jusqu'à présent, et dont a été retrouvé un seuil en calcaire. Le tout était recouvert de cette épaisse couche de destruction repérée ailleurs. À signaler, deux petits objets donnant une date : une lampe du type $\mathrm{X}$ de Corinthe (fin IIIe-début Ier s. av. J.-C.) et une monnaie de Patras (entre 146 et 32 av. J.-C.). Au nord de la pièce avec foyer on distingue encore au moins deux pièces avec une cuvette carrée, de petites dimensions, comparable à celle de la pièce avec petite baignoire découverte l'année dernière, appartenant sans doute comme celle-ci à la deuxième phase de l'occupation. Audessus, une couche de destruction et un troisième niveau d'habitat.

Des fragments de lampes du type XXVII de Corinthe (deuxième moitié du IIe s. av. J.-C.) confirment aux yeux du fouilleur l'hypothèse formulée l'année dernière, à savoir que les deux principales phases de construction dans la région ont été interrompues par une catastrophe qui a été suivie, plus tard, d'une nouvelle occupation lors de laquelle certains endroits ont été reconstruits tandis que des anciens ont été remis en utilisation, mais lentement.

\section{Activités de l'Institut canadien d'archéologie}

\section{Eubée}

"Canadian Southern Euboea Exploration Project" (M. Wallace D.Keller)

Achèvement de l'étude du matériel collecté durant les campagnes précédentes et conservé dans les réserves du musée de Karystos, et prospection sur la péninsule de Paximadhi. Kirsten Gay poursuit ses recherches sur les sanctuaires ruraux de la péninsule.

\section{Lesbos}

a. Moria, village à quatre $\mathrm{km}$. au nord de Mytilène $(H$. Williams University of British Columbia)

Prospection d'une importante carrière de marbre d'époque romaine, qui conserve encore des centaines de membres architecturaux ainsi que des bassins et des autels à différents stades d'exécution. 


\section{b. Mytilène (H. Williams - University of British Columbia)}

Préparation de la publication finale des fouilles au port nord et dans le sanctuaire de Déméter sur l'Acropole. Les résultats de l'étude faunique sont notables. En plus du fait que l'abondance du matériel (plus de 60.000 ossements) et son échelonnement sur une période très étendue (du VIIe s. av. J.-C. au début du XIXe s. ap. J.-C.) ont permis de procéder à une étude diachronique à une échelle rarement atteinte sur un site grec, l'étude des ossements provenant du sanctuaire de Déméter a révélé qu'à côté de ceux de cochons et de porcelets, il y en avait de chiens qui portaient des marques de boucher.

\section{Crète}

«Sphakia Survey» (L. Nixon, University of New Brunswick at Saint John, J. Moody, Baylor University)

Durant cette saison, c'est d'abord un important travail de terrain qui a été réalisé : visite et découverte de 81 sites, portant à 218 le nombre de sites enregistrés. La région de Madares, dans les montagnes Blanches, a été plus particulièrement étudiée et réunit à elle seule 18 sites, dont les plus anciens remontent au néolithique final-minoen ancien et dont certains autres vont jusqu'à l'époque romaine. Il en est un qui, par l'altitude à laquelle il est situé (1810-1840 m), est estimé être un des plus grands sites du MM-MR les plus élevés de Crète.

\section{Activités de l'Institut norvégien d'archéologie}

Tégée. Sanctuaire d'Athéna Aléa (Erik Østby)

C'était la troisième saison du programme de fouille, qui réunit dans une étroite collaboration l'Institut norvégien, l'Institut suédois et les Écoles française et italienne d'archéologie, ainsi que l'Université de l'Arizona (USA) et les archéologues grecs Th. Spyropoulos et A. Delivorrias.

Dans le secteur aligné sur la rampe d'entrée nord du temple, la surface du sanctuaire en usage depuis la construction du temple jusqu'à la fin de l'antiquité a été atteinte. Il semble que ce secteur ait servi de cour ouverte, dépourvue de constructions ou d'autres monuments, bien qu'il y ait des édifices alentours. Le sol, pourtant exceptionnellement bien conservé, est rayé en un endroit par les ornières laissées par un chariot d'une grandeur et d'un poids inhabituels.

Dans le carré contigu, les couches archaïques sous la surface de la cour ont été atteintes. Dans la première de celles-ci, une série de trous de 
poteaux suggère l'existence d'une structure légère, en bois, de dimensions et de forme incertaines.

Près du temple, les deux bases de monument de l'époque classique que les fouilles françaises de 1910 avaient découvertes sont à nouveau exposées. La fouille du temple entre les fondations de la colonnade intérieure du temple archaïque ancien s'est poursuivie et a révélé, près de la fondation archaïque du nord, l'existence d'un édifice cultuel un peu plus grand et probablement un peu plus tardif. Cet édifice semble avoir la même forme absidiale que son prédécesseur, et avoir été construit avec la même technique basée sur un système de poteaux verticaux à l'intérieur et à l'extérieur, sans socle de pierre. Il y a peut-être aussi des traces d'une banquette intérieure, le long du mur nord. Le matériel qui provient de cette construction date du Géométrique récent et se laisse très difficilement distinguer pour l'instant du matériel à rapporter à l'édifice plus ancien.

Dans la région du pronaos du temple classique, un secteur qui a échappé aux anciennes fouilles françaises a livré une fosse remplie de terre et de scories métalliques, associées à de la céramique du Géométrique Moyen à Récent. Ceci indique, aux yeux du fouilleur, une activité métallurgique dans le sanctuaire Géométrique.

Les couches des deux édifices cultuels Géométriques ont donné des restes considérables d'ossements de sacrifices et de repas rituels, lesquels vont faire l'objet d'une étude minutieuse visant à reconstituer le rituel.

Dans les couches d'époque classique du secteur nord on a identifié une dent de lion. 